\title{
A CASE OF INTENSIVE THERAPY OBTAINED A FAVORABLE QUALITY OF LIFE FOR ADVANCED GASTRIC CANCER WITH DISTANT METASTASIS
}

\author{
Ryozo Iwazaki, Hiroto Miwa, Toshio Yamada, Koichi Kashiwakura, Katsuyori Iijima \\ Yasuhiko Ohno, Kazuki Ohta', Motomichi Urabe ${ }^{2}$, Toshiharu Matsumoto ${ }^{3}$ \\ Nobuhiro Sato ${ }^{\text {l* }}$
}

\author{
Department of Gastroenterology ${ }^{1}$, First Department of surgery ${ }^{2}$ \\ and First Department of pathology ${ }^{3}$ \\ Juntendo University School of Medicine \\ Hongo 2-1-1, Bunkyo-Ku, Tokyo, 113-8421, Japan \\ TEL:+81-3-3813-3111; FAX:+81-3-3813-8862
}

\begin{abstract}
Chemotherapy administered to a case of non-resectable advanced gastric cancer with esophageal invasion and bone metastasis was found to be remarkably effective over two years and 4 months. For imaging investigation, the efficacy of the chemotherapy was judged to be a partial response (PR), Furthermore, DNA histograms prepared by flow cytometry as a diagnostic test for malignancy of cancer revealed changes in the primary lesion from an aneuploidy pattern to a diploidy pattern concomitant with the administration of chemotherapy. Since non-phasic metastasis to the cerebellum and the adrenals were detected subsequently, intensive therapy consisting of radical gastric resection, left adrenal resection and deep cerebellur nucleus resection combined with radiotherapy, was conducted with the objective of prolonging the survival of the patient. The total course extended for about 4 years and considering the stage of advanced cancer, we believe that a favorable quality of life (QOL) was achieved based on the Performance status(P.S) level, maintenance of a satisfactory level of meal consumption, continuation of employment at times other than hospital admission and a home-residence rate of 72.3 per cent.
\end{abstract}

\section{Introduction}

The effectiveness of chemotherapy for non-resectable gastric cancer accompanied by distant metastasis is poor (MacDonalds, Philip et al., 1980; Wolly, Smithet al., 1981; Preusser, Wilke et al., 1987). In addition, various other associated problems, such as long-term hospital admission and Performance Status reduction, impose difficulties on

\footnotetext{
*Correspondence: Professor Nobuhiro Sato, Department of Gastroenterology, Juntendo University School of Medicine, Hongo 2-1-1, Bunkyo-ku, Tokyo, 113-8421, Japan. TEL:+81-3-3813-3111; FAX:+81-3-3813-8862
} 
the achievement of a favorable quality of life (QOL) (Takahashi and Nishioka, 1995). However, the only criteria for evaluating the efficacy of chemotherapy are the findings of imaging investigations, which do not reflect the degree of malignancy of cancer, and the judgment of chemotherapy efficacy and the survival time are not necessarily correlated. We experienced a case in whom chemotherapy was effective not only in terms of tumor imaging but also in favorable QOL with satisfactory long-term levels of P.S, meal intake and a home residence rate and flow cytometric analysis of DNA histogram (Sasaki and Ogino, 1986; Danova, Mazzini et al., 1987) obtained from the tumor tissue showed improvement of the degree of malignancy as the improvement of the disease.

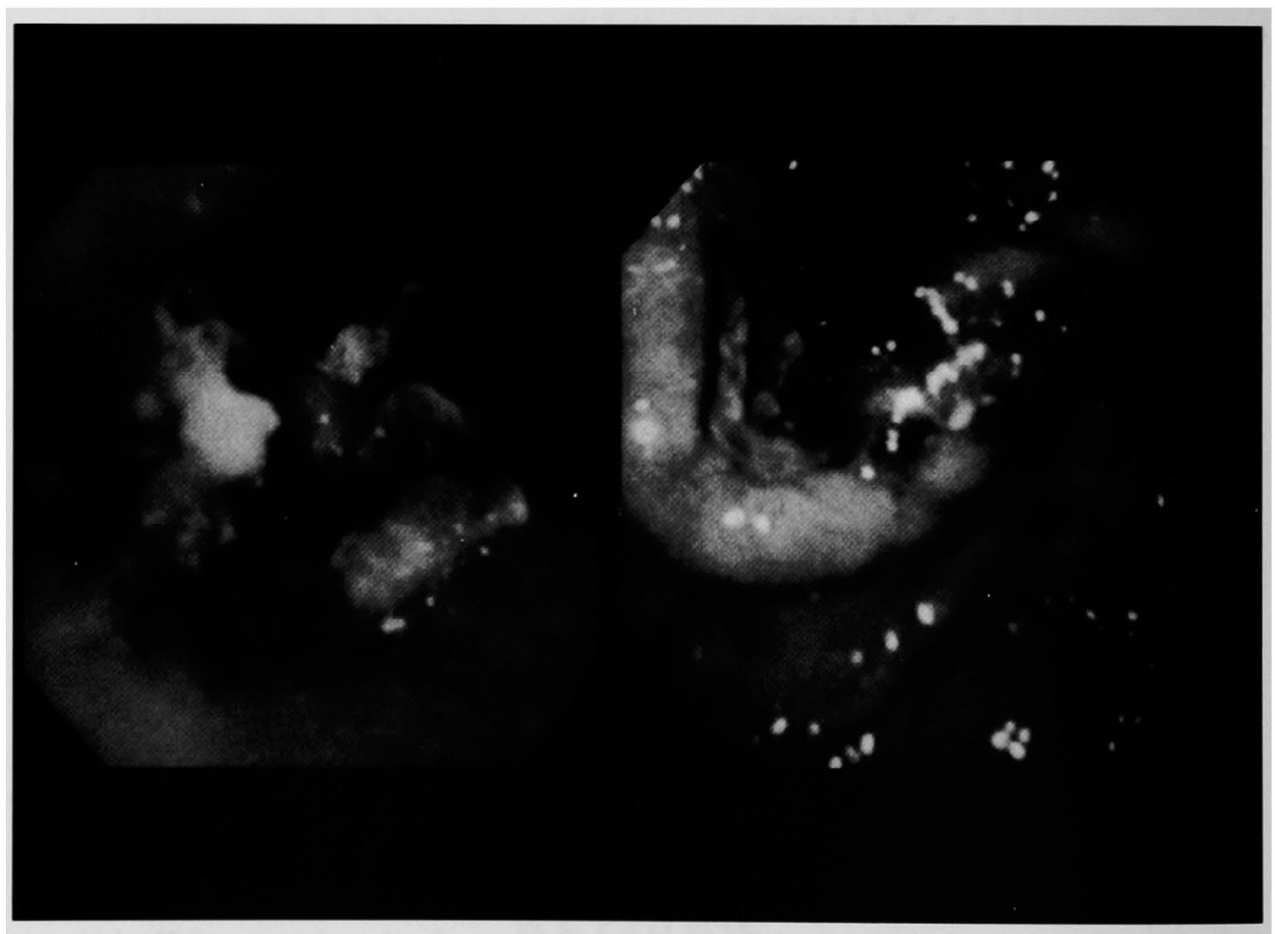

Figure 1a: Endoscopic picture showing Type 2 advanced gastric cancer in the cardia and invasion to the esophagus.

\section{Case Report}

The patient was a 38-year-old man who had dyspeptic symptoms after eating. Gastric cancer of the cardia was presumptively detected by barium meal study at a medical check 
VOL. 9, NOS. $1 \& 2$

ANNAILS OIF

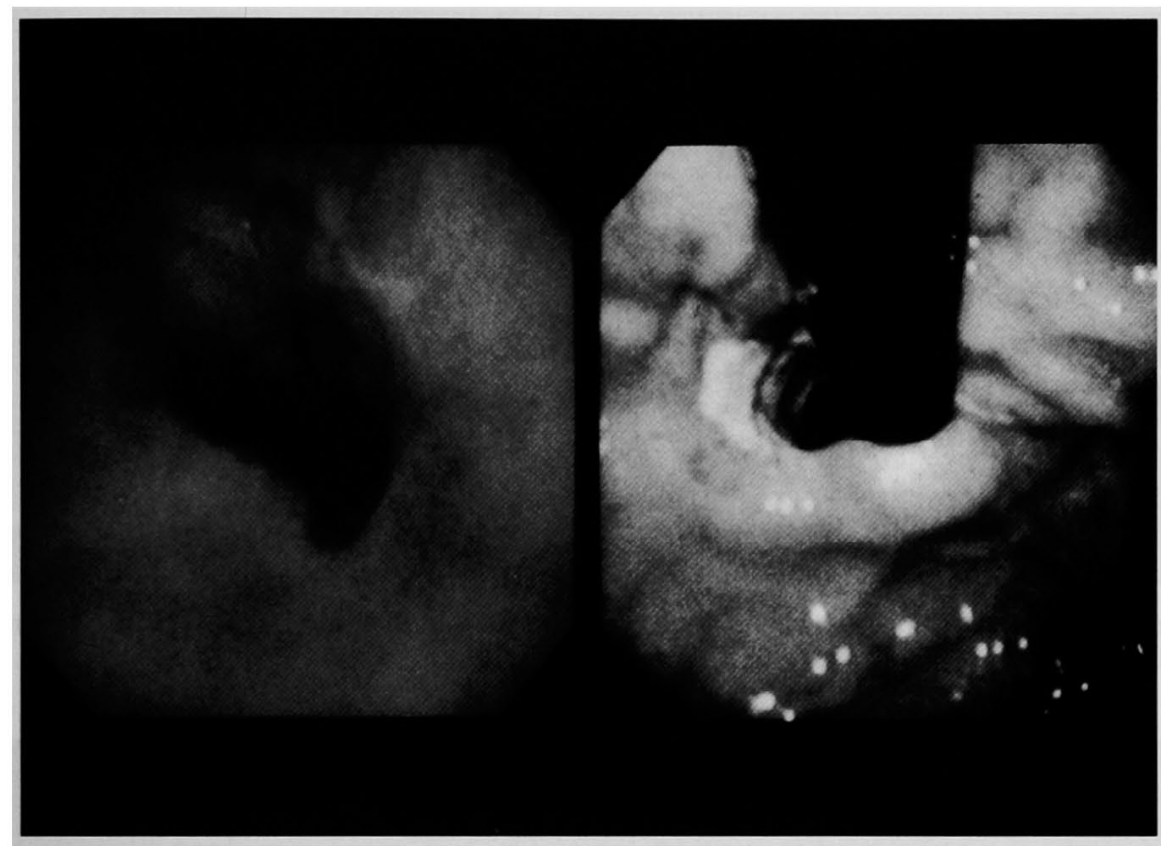

Figure $1 b$ : Type 2 advanced gastric cancer was remarkably reduced and the esophageal lesion had disappeared after 2 courses of UFTMP therapy.

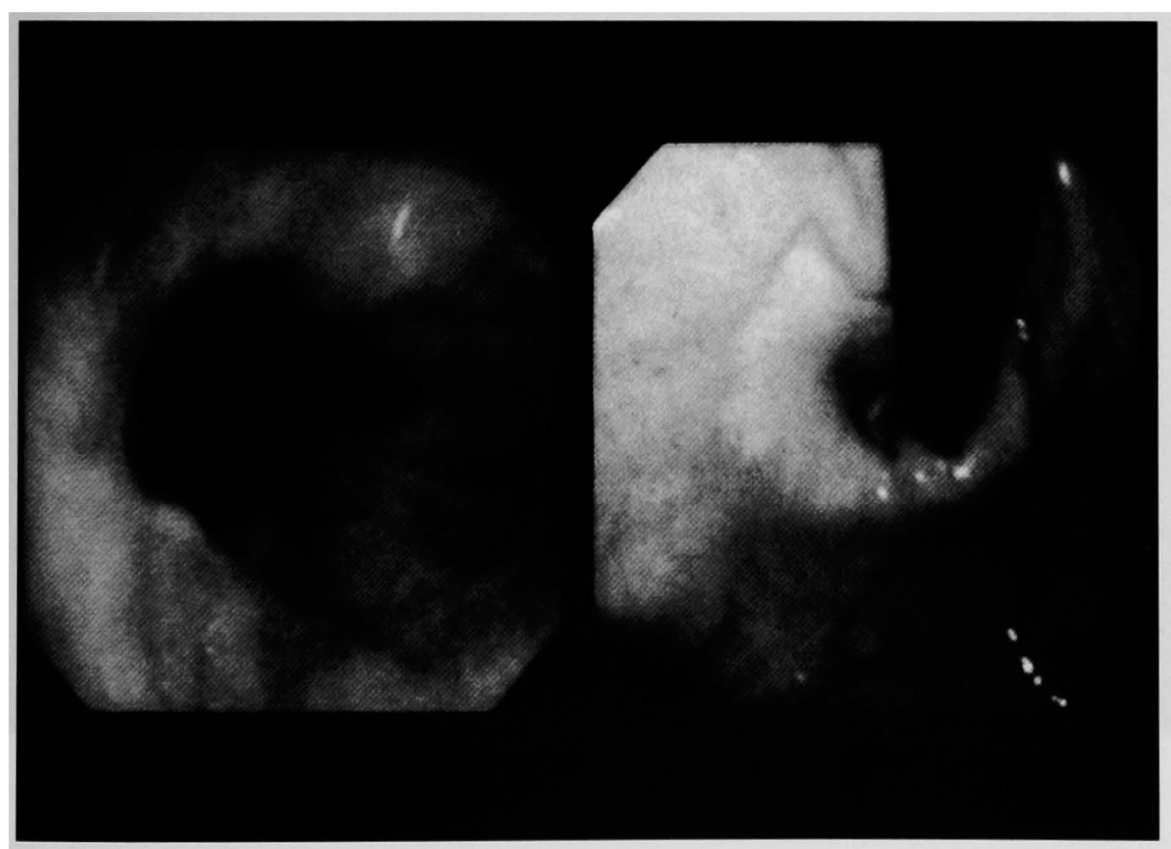

Figure 1c: Type 2 advanced gastric cancer was remarkably reduced and the esophageal lesion had disappeared after 4 courses of UFTMP therapy. 
VOL. 9, NOS. $1 \& 2$ 2001
ANNALS OIF Cancer Research and Therapy

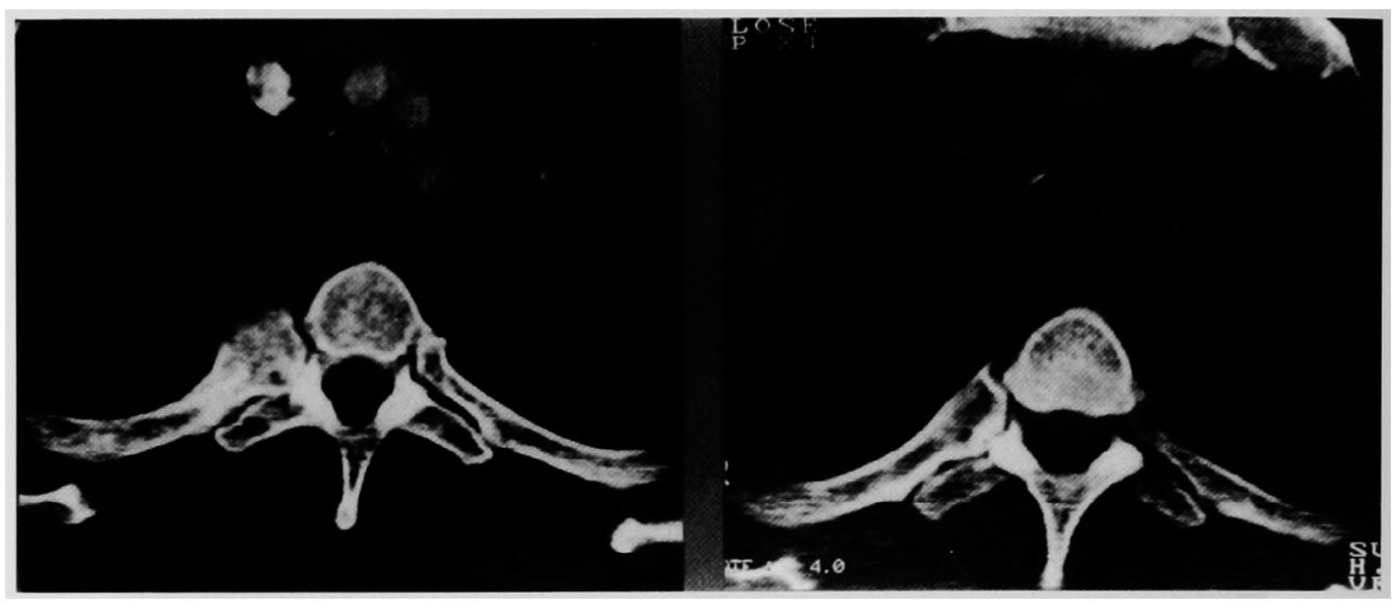

Figure 2a: Thoracic CT showing metastatic tumor of the right fourth rib. (b): Metastatic tumor had disappeared after 2 courses of UFTMP therapy.

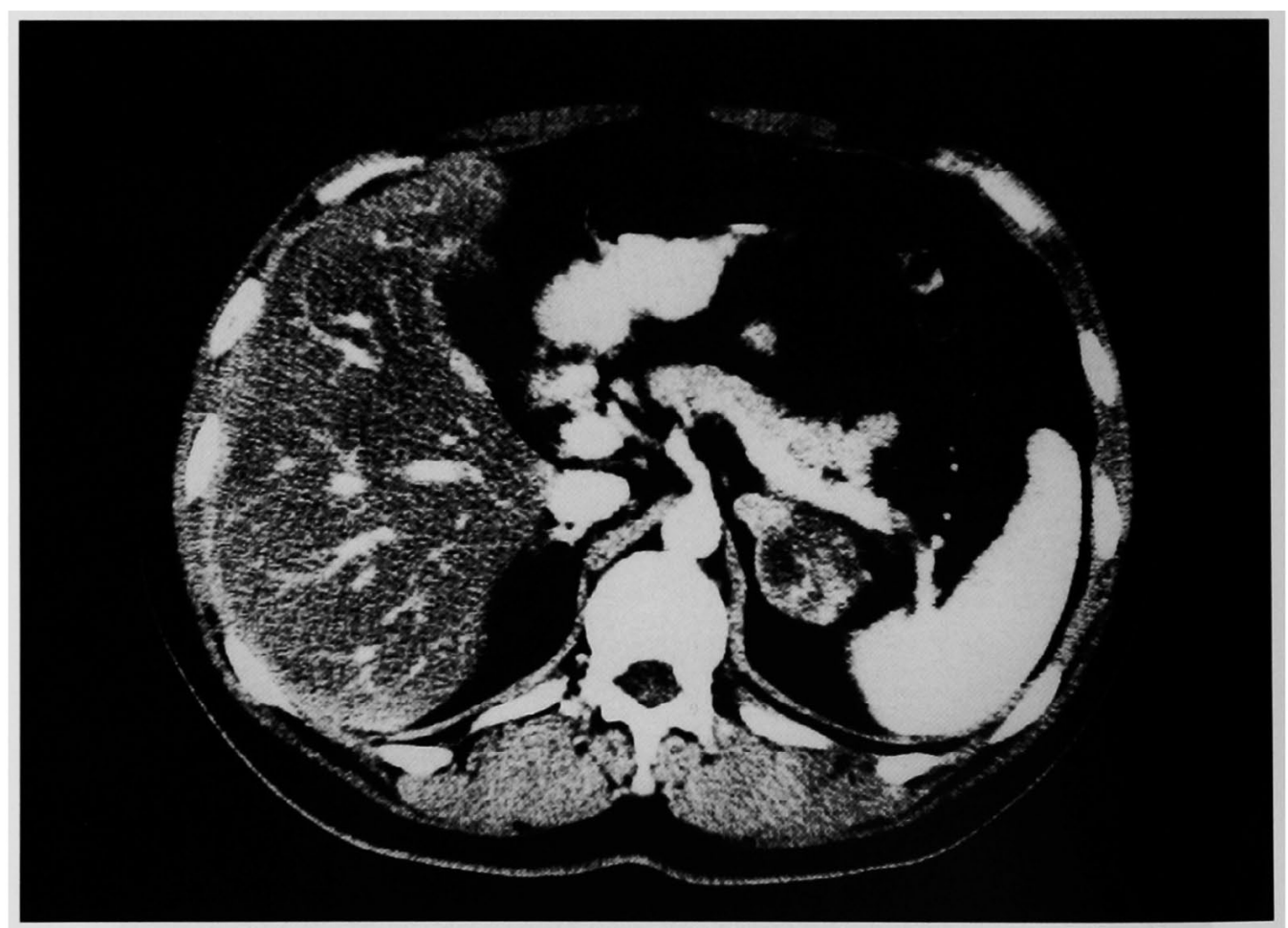

Figure 3: Abdominal CT showing metastatic tumor in the left adrenal area. 
VOL. 9, NOS. $1 \& 2$

2001
ANNAULS OIF

Cancer Research and Therapy

Clinical course

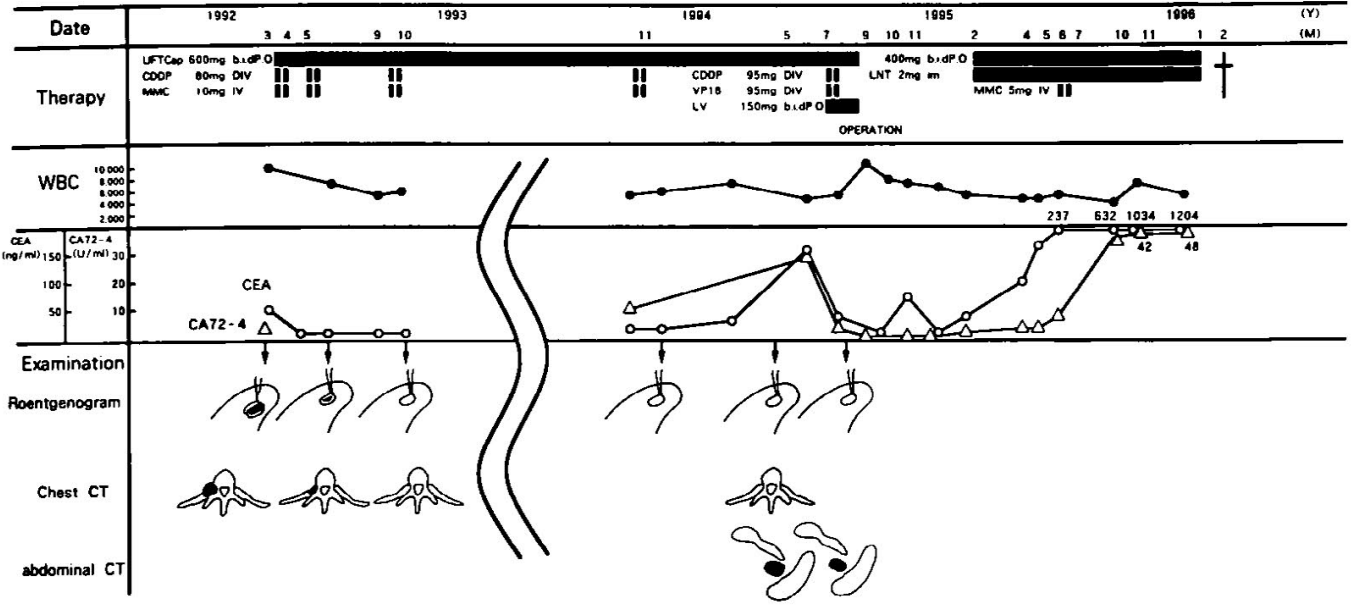

Figure 4: Clinical course.

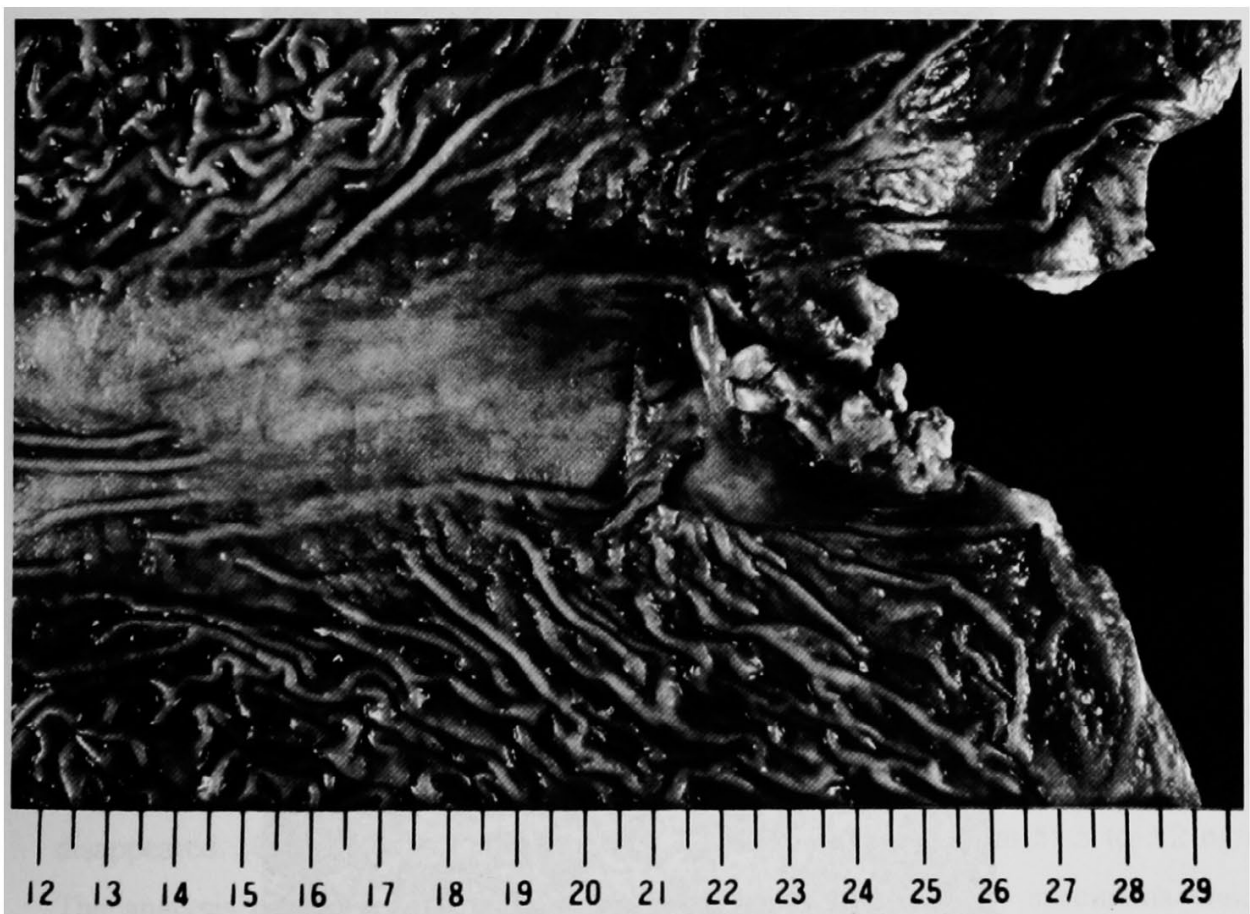

Figure 5: Macroscopic picture of resected stomach after chemotherapy. 
VOL. 9, NOS. 1 \& 2

2001

\author{
ANNALS OF \\ Cancer Research and Therapy
}

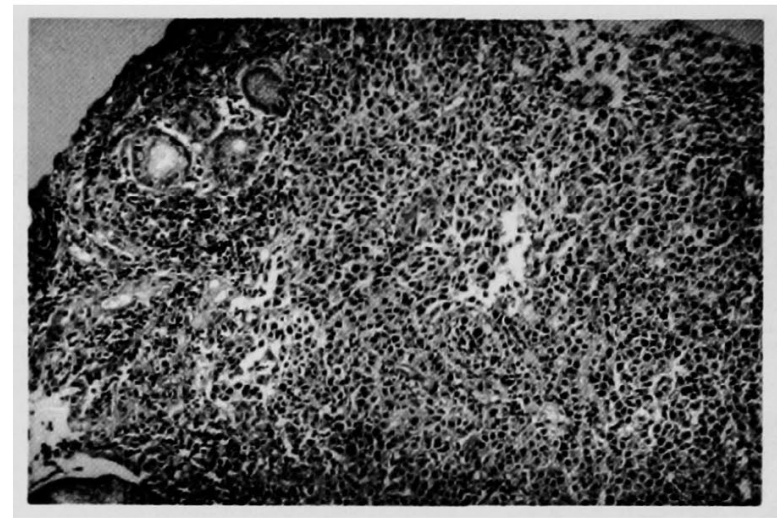

Figure 6a: Histopathological picture of biopsy specimen before chemotherapy. Pathology of the Type 2 advanced gastric cancer was poorly differentiated adeno-carcinoma.

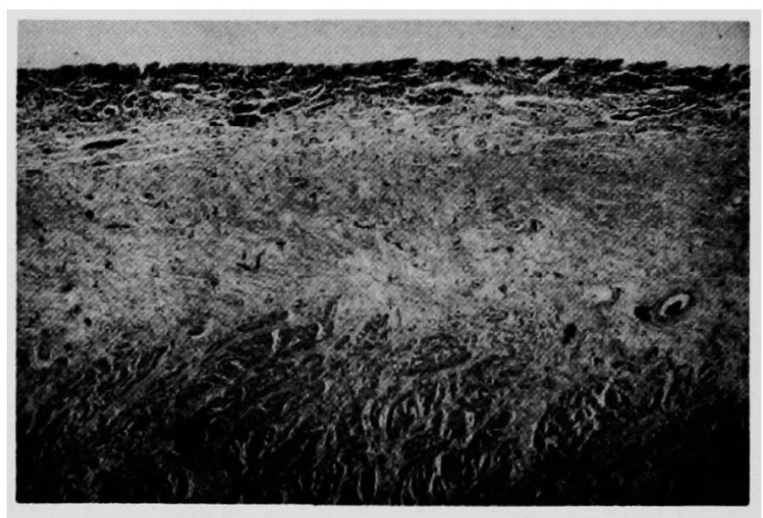

Figure $6 b$ : Histopathological picture of resected stomach after chemotherapy. Cancer cells from the lesion of the gastric resection could not be detected by histopathological examination.

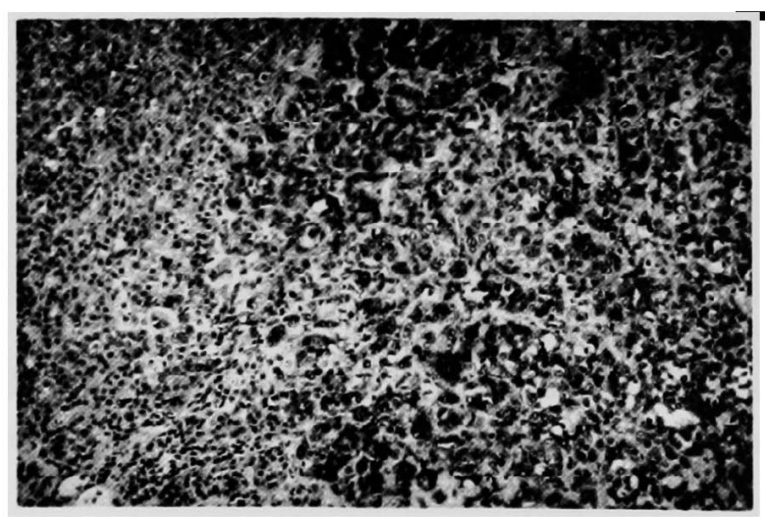

Figure 6c: Histopathological picture of resected left adrenal after chemotherapy. Poorly differentiated cancer cells similar to those of the primary lesion were observed in the left adrenal. 


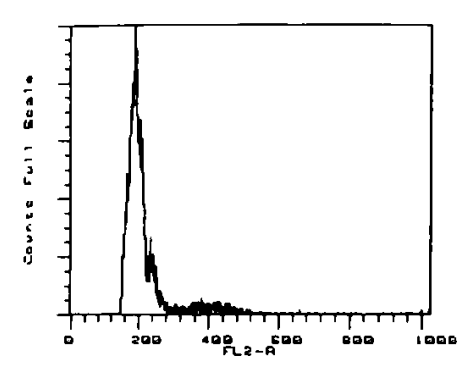

Figure 7a

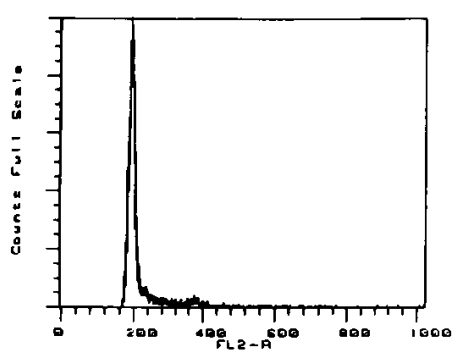

Figure 7b

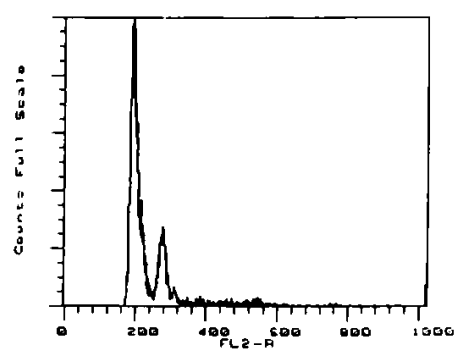

Figure $7 \mathrm{c}$

Figure 7a: DNA histogram showing an aneuploidy pattern from biopsy specimens of gastric cancer before systemic chemotherapy. (b): DNA histogram showing a diploidy pattern from biopsy specimens and resected specimens of gastric cancer after systemic chemotherapy. (c): DNA histogram showing an aneuploidy pattern from resected specimens of tumor mass of adrenal gland after systemic chemotherapy.

up in March 1992, after which the patient was referred to our hospital. After admission, and upper gastrointestinal endoscopy (Figure 1a) demonstrated type 2 advanced gastric cancer of the cardia, which had extended to the esophagus. Biopsy specimens obtained from the tumor histologically revealed a poorly differentiated adenocarcinoma. In addition, thoracic CT (Figure 2a) demonstrated bone metastasis to the right fourth rib. After informed consent was obtained from the patient's family in April, he was administered UFTMP therapy [UFT (Uracil-Tegafur): cap $400 \mathrm{mg} / \mathrm{m}^{2}$ p.o on days 1-28; MMC (Mitomycin C): $5 \mathrm{mg} / \mathrm{m}^{2} \mathrm{IV}$ on days 1 and 8; CDDP (Cisplatin): $50 \mathrm{mg} / \mathrm{m}^{2} \mathrm{DIV}$ on days 1 and 8] (Iwazaki, Yasutakeet al., 1993). After undergoing 2 courses of this systemic chemotherapy, the primary lesion that had been originally $6.5 \times 3.5 \mathrm{~cm}$ was reduced to $4.5 \times 2.5 \mathrm{~cm}$ (Figure $1 \mathrm{~b}$ ). In addition, not only the esophageal lesion but also the rib metastasis had disappeared (Figure 2b). Accordingly, the efficacy of the chemotherapy was judged to be a partial response (PR) for primary lesion had not disappeared. Carcino embryonic antigen (CEA) had decreased from 59.5 to $3.2 \mathrm{ng} / \mathrm{ml}$. The analysis of degree of malignancy of the tumor using biopsy specimens yielded negative immunostaining results for the oncogene, $\mathrm{p} 53$. Moreover, the DNA histogram obtained by flow cytometry revealed an aneuploidy pattern had changed to a ploidy pattern in the course of the chemotherapy. Two more courses of the same chemotherapy 
were administered to maintain the disease status. During this period, adverse effects such as nausea, vomiting and appetite loss were not observed, and data of blood chemistry analysis remained in the normal range.

TABLE I

Laboratory data on admission

Peripheral blood

$\begin{array}{cr}\text { WBC } & 5400 / \mu l \\ \text { RBC } & 506 \times 10^{\circ} / \mu \ell \\ \mathrm{Hb} & 16.2 \mathrm{~g} / \mathrm{d} \ell \\ \mathrm{Ht} & 46.6 \% \\ \text { PLT } & 17.8 \times 10^{3} / \mu \mathrm{l}\end{array}$

Blood chemistry

$\begin{array}{lc}\text { ALP } & 195 \mathrm{lU} / \ell \\ \text { GOT } & 26 \mathrm{lU} / \ell \\ \text { GPT } & 30 \mathrm{lU} / \ell \\ \gamma \text {-GTP } & 35 \mathrm{U} / \ell \\ \text { LAP } & 164 \mathrm{GRU} \\ \text { LDH } & 392 \mathrm{lU} / \ell \\ \text { TBIL } & 1.2 \mathrm{mg} / \mathrm{d} \ell \\ \text { DBIL } & 0.2 \mathrm{mg} / \mathrm{d} \ell \\ \text { CHE } & 1140 \mathrm{lU} / \ell \\ \text { T-CHO } & 261 \mathrm{mg} / \mathrm{d} \ell \\ \text { TG } & 1098 \mathrm{mg} / \mathrm{d} \ell \\ \text { BUN } & 12 \mathrm{mg} / \mathrm{d} \ell \\ \text { CRE } & 0.9 \mathrm{mg} / \mathrm{d} \ell \\ \mathrm{Na} & 139 \mathrm{mM} / \ell \\ \mathrm{K} & 4.3 \mathrm{mM} / \ell \\ \mathrm{Cl} & 103 \mathrm{mM} / \ell \\ 24 \mathrm{Ccr} & 97.6 \mathrm{~m} \ell / \mathrm{min}\end{array}$

$17.8 \times 10^{3} / \mu 2$

\begin{tabular}{lrl} 
Serum & & \\
\hline AFP & 7 & $\mathrm{ng} / \mathrm{ml}$ \\
CEA & 166.8 & $\mathrm{ng} / \mathrm{ml}$ \\
\hline CA19-9 & 74 & $\mathrm{U} / \mathrm{ml}$ \\
\hline CA72-4 & 32 & $\mathrm{U} / \mathrm{ml}$
\end{tabular}


and the heart rate was $68 / \mathrm{min}$. He had no symptoms such as anemia or icterus of the conjunctivae, swelling of the superficial lymph nodes or hepatomegaly. No abnormalities were found in the peripheral blood and by blood biochemistry analysis, although CEA, CA72-4, and carbohydrate antigen 19-9 (CA19-9) levels were elevated. Adrenal function test revealed slight elevation of urine 17-OHCS (Table I). Upper gastrointestinal endoscopy (Figure 1c) also showed a shallow depression that suggested a cType 0 IIc T1-like lesion of $4.5 \times 2 \mathrm{~cm}$ at the cardia of the stomach. However, cancer cells were histologically not present on the biopsy specimens obtained from the gastric lesion. Bone scintigraphy to investigate the recurrence of bone metastasis showed negative results. Abdominal CT (Figure 3) demonstrated a $3.0 \times 1.8 \mathrm{~cm}$ tumor image corresponding to the left adrenal, confirming the diagnosis of left adrenal metastasis. Rib metastasis, enlarged lymph nodes, liver metastasis and ascites were not observed. Time of recurrence is shown in the schematic chart of his clinical course (Figure 4). For the recurrence, 1 course of systemic chemotherapy by FLEP [(UFT: cap $400 \mathrm{mg} / \mathrm{m}^{2}$ p.o on days $1-28 ; \mathrm{LV}$ (Leucovorin): $100 \mathrm{mg} / \mathrm{m}^{2}$ p.o on days 1-28; VP16 (Etoposhide): $50 \mathrm{mg} / \mathrm{m}^{2}$ DIV on days 1 and 8; CDDP:50mg/m ${ }^{2}$ DIV on days 1 and 8)] was begun on July 25, 1994. Although the new chemotherapy was effective for decreasing the levels of CEA, CA72-4, and CA19-9, the size of the left adrenal gland tumor remained the same. Accordingly, the patient underwent total gastrectomy and extirpation of the left adrenal gland on September 20, 1994, at the First Department of Surgery of our hospital. Surgical laparotomy revealed that the stage of the cancer was sType 2 T3 (SS) HOPONOMOD3, stage II, and the surgery was designated as radical cure $B$ according to Japanese Classification of Gastric Carcinoma (The 13th Edition, 1999). Macroscopic findings of the resected gastric lesion (Figure 5) showed a shallow depression, suggesting a Type 0IIcT1 lesion with $4.5 \times 2.0 \mathrm{~cm}$ in size located at the cardia of the stomach. The extirpated left adrenal gland was coalescent with the kidney. No cancer cells were detected on the gastric lesion or the resected lymph nodes by histopathological examination (Figures $6 \mathrm{a}, 6 \mathrm{~b}$ ) following the operation. The stage of the disease was diagnosed as Grade 3 based on the criteria of histopathological evaluation of chemotherapy for gastric cancer. However, histological examination of the tumor mass 
of the adrenal gland revealed poorly differentiated adenocarcinoma cells similar to those of the primary lesion (Figure 6c). DNA histogram obtained by flow cytometry revealed again an aneuploidy pattern that had undergone transition to a diploidy pattern by the first chemotherapy, and tumor tissue from adrenal gland also showed aneuploidy pattern (Figures $7 \mathrm{a}, 7 \mathrm{~b}, 7 \mathrm{c}$ ). The patient had been scheduled to be discharged from hospital in November 1994.

However, the patient began to manifest a severe headache, and the values for the tumor marker, CEA, increased again, from 10.8 to $46.3 \mathrm{ng} / \mathrm{ml}$. As the brain CT performed at that time revealed a small brain metastasis, his discharge was postponed and he underwent intensive therapy, including tumor extirpation and radiotherapy at the Neurosurgery and Radiology Departments of our hospital. Regardless of such therapies, he died of cachexia 18 months after the surgical laparotomy (survival time 1420 days).

\section{Discussion}

Non-resectable advanced gastric cancers accompanied by distant metastasis are treated by a variety of therapies, including systemic chemotherapy with combined multiple drugs (MacDonalds, Philip et al., 1980; Wolly, Smith et al., 1981; Preusser, Wilke et al., 1987) and immunotherapy (Mizumoto, Ohoue et al., 1989). However, outcome in such diseases has not been still satisfactory. There has been a recent trend in Japan toward an increase of cases in whom systemic chemotherapy for gastric cancer was effective to some extent. Nevertheless, there have been few reports Nakata, Shigemitsu et al., 1985; Ono, Ishikawa et al., 1994; Tsuji, Suematsu et al., 1994; Iwazaki, Okura et al., 1994; Iwazaki, Maehiro et al., 1996) documenting remarkable effectiveness of systemic chemotherapy in advanced gastric cancers. Moreover, complete response to the chemotherapy was not histologically confirmed in most of such cases. As to patients showing marked improvement of advanced gastric cancer by chemotherapy (Kurihara, Izumiet al., 1977; Tokimatsu, Yasutake et al., 1989) have reported that such results had to be seen in [1] patients aged 70 years or older, [2] patients administered Tegaful as the main carcinostatic drug, [3] patients with type 2 gastric cancer without distant metastasis, and [4] patients with a tumor that was a histopathologically differentiated type of 
adenocarcinoma. In other words, these findings suggest that the combined use of 5-FU and its derivatives may be effective for gastric cancer with histologically well-differentiated type without distant metastasis.

In our case, patients of 38 years old was in good general condition, type 2 gastric cancer with esophageal invasion and bone metastasis, and that the patient with a tumor that was a histopathologically poorly differentiated type of adenocarcinoma. However, the primary cancer and metastatic lesions were found to be remarkably decreased in size and denatured by chemotherapy (UFTMPtherapy) (Iwazaki, Yasutake et al., 1993) of two courses.

At the present time, since the efficacy of chemotherapy are evaluated only by morphological of images investigations that do not reflect the biological malignant degree of tumors, efficacy of chemotherapy and the survival time (Takahashi and Nishioka, 1995) are not necessarily closely correlated. The rate and capacity of proliferation of a cancer are considered to be predicting factors for biological malignant degree. Recently, immunostaining technique (Sasaki and Ogino, 1986; Danova, Mazzini et al., 1987; Hoshino, Prados et al., 1989; ROBBINS, Vega et al., 1987; Ploton, Menager et al., 1986; Crocker, 1990;Gerdes, Lemke et al., 1984; Lowe, Bodis et al., 1994; James, Richard et al., 1986; Korenaga, Okamura et al., 1988; Olga and Galina, 1998; Saito, Korenaga et al., 1991) that employs an antibody specific to an antigen (proliferating cell antigen), and is closely associated with the cell proliferation activity, have become available for predicting factors for proliferative capacity of a tumor. These markers includes BrdU (bromodeoxyuridine) (Hoshino, Prados et al., 1989), PCNA (proliferating cell nuclear antigen) (Robbins, Vega et al., 1987), AgNORs (Ploton, Menager et al., 1986; Crocker, 1990), Ki67 (Gerdes, Lemke et al., 1984), DNA histograms produced by flow cytometry (Sasaki and Ogino, 1986; Danova, Mazzini et al., 1987) or oncogenes, such as p53 (Lowe, Bodis et al., 1994).

The biological features of gastric cancer are considered to vary in accordance with tissue type. Differentiated cancers often demonstrate marked vascular infiltration leading easily to hematogenic metastasis and poorly differentiated cancers demonstrate high cell proliferative activity leading easily to lymph node metastasis and peritoneal implantation. 
Analysis of the degree of malignancy of gastric cancer using a DNA histogram obtained by flow cytometry has shown that an aneuploidy DNA pattern is common in tumors that show a high level of cell proliferative activity, frequent lymph node metastasis and an especially marked tendency for deep-site infiltration (James, Richard et al., 1986; Korenaga, Okamura et al., 1988; Olga and Galina, 1998). In aspect of the tissue type of gastric cancers, it has been shown that there is a tendency that undifferentiated types have a rather higher level of susceptibility to anti-cancer agents than differentiated types (Saito, Korenaga et al., 1991). It has also been reported that poorly differentiated types with an aneuploidy pattern show a higher level of susceptibility to anti-cancer agents than differentiated types by analyzes of DNA histograms obtained by flow cytometry (Maehara, Anai et al., 1987).

In our case, biopsy specimens yielded negative immunostaining results for the oncogene, p53. Accordingly, we considered that p53 was normal, and we could not technically detect a mutants of p53. The DNA histogram obtained by flow cytometry in this case revealed an aneuploidy pattern, which had shown a diploidy DNA pattern concomitant with the course of remarkably effective chemotherapy. As a result, in spite of the fact that this was a case of non-resectable advanced gastric cancer, the treatment was remarkably effective with a favorable QOL (Takahashi and Nishioka, 1995) evaluated on the basis of long-term satisfactory P.S, meal intake levels, high rate of home residence for 2 years and a relatively long period until the appearance of adrenal metastasis.

Intensive therapy include chemotherapy should be performed with a case of this type. This active strategy may enable a better outcome to be achieved not only in terms of tumor imaging, but also in favorable QOL with satisfactory long-term levels of P.S, meal intake and a home residence rate.

In the future, we have to randomize comparisons of phase III study of chemotherapy with the use of best supportive care (BSC) alone in patients with non-resectable gastric cancer (Glimelius, 1994; Pyrhonen, 1995). 


\section{References}

Crocker, J. (1990). Nucleolar Organizer Regions. Pathology of the nucleus (Underwood, J.C.E et.), Springer-Verlag :91-149.

Gerdes, J., Lemke, H., Baisch, H. (1984). Cell cycle analysis of a cell proliferationassociated human nuclear antigen defined by the monoclonal antibody Ki-67. J. Immunol. 133:1710-1715.

Glimelius, B. (1994). Initial or delayed chemotherapy with best supportive care in advanced gastric cancer. Ann. Oncol. 5:189-190.

Hoshino T, M.Prados, C.B., Wilson, K.G., Lee, R.L., Davis. (1989). Prognostic implications of bromodeoxyuridine labeling index (BrdU-LI) in human gliomas. J. Neurosurg. 71:335-341.

Iwazaki R, Yasutake K. (1993). UFTPM(UFT+MMC+CDDP) Therapy for progressive stomach cancer. Jpn.J.Cancer Chemother. 20:1179-1185.

Iwazaki R, Okura R, Yamada T, Kobayashi O, Terai T, Watanabe H, Sung Wook Yang, Ogihara T, Yamada S, Sato N, Kondo K, Hamada T. (1994). A case of multiple gastric cancer with complete se of response of advanced cancer and liver metastasis, but no response of early cancer chemotherapy. J.Jpn.Soc.Cancer Ther. 29:1921-1926.

Iwazaki R, Maehiro K, Iijima K, Ota K, Ono Y, Sato K, Okura R, Miwa H, Sato N, Mizobuchi N, Urabe M and Wada R. (1996). A case of advanced gastric cancer remarkably responding preoperative UFT.E therapy. Jpn.J.Cancer Chemother. 23:355-359.

James C. Macartney, Richard S. Camplejohn, Gillian Powell. (1986). DNA Flow Cytometry Of Histological Material From Human Gastric Cancer. J. Pathology 148:273-277.

Japanese Classification of Gastric Carcinoma (The 13th Edition, 1999). Japanese Gastric Cancer Association 1-106.

Korenaga D, Okamura T, Saito A. (1988). DNA ploidy is closely linked to tumor invasion, lymph node metastasis and prognosis in clinical gastric cancer. Cancer 62:309-313.

Kurihara M, Izumi T, Miyasaka K, Shirakabe H. (1977). Improvement of radiological, endoscopic and biopsied findings of gastric cancer by anti-cancer chemotherapy. Jpn.J.Cancer Chemother. 330-336.

Lowe,S.W., Bodis, S., McClatchey, A .(1994). p53 status and the efficacy of cancer therapy in vivo. Science 266: 807-810.

MacDonalds, J.S., Philip, S.Schein., Paul, V. Wolley. (1980). 5-Fluorouracil, doxorubicin, and mitomycin C (FAM) combination chemotherapy for advanced gastric cancer. Ann.Int.Med. 93: 533-536.

Maehara Y, Anai H, Kusumoto H. (1987). Poorly differentiated human gastric carcinoma is more sensitive to anti-tumor drugs than is well differentiated carcinoma. Eur.J.Surg.Oncol. 13: 203-206.

M.Danova, G.Mazzini, G.Wilson, G.Ucci, P.Dionigi, A.Riccardi, and R.Fiocca. (1987). Ploidy and proliferative activity of human gastric carcinoma cytofluorometric study on fresh and on paraffin embedded material. Basic Appl. Histochem. 31:73-82. 
Mizumoto S, Ohoue M, Takami Y, lihara K, Kurokawa E, Akashi H, Aoki Y, Mori H, Okano K. (1989). An autopsied case of an aged patient with an advanced gastric cancer that showed a complete response to immuno-chemotherapy. Jpn.J.Cancer Clin. 35: 1791-1794.

Nakata K, Shigemitsu M. (1985). Two cases of advanced gastric cancer which responded to a combined chemotherapy with UFT and mitomycin C. Jpn. J. Cancer Chemother. 12: 1694-1696.

Olga V. Chistyakova, Galina N. Zubrikhina. (1998). Flow Cytometric Analysis of DNA Content in Gastric and Colorectal Cancer. Anal.Quant.Cytol.Histol. 20: 52-58.

Ono, M, Ishikawa H, Suzuki N, Miura T, Kaga S, Kano A. (1994). A case of gastric cancer completely responding to sequential methotrexate and 5-fluorouracil therapy. Jpn.J.Cancer Chemother. 21: 2035-2038.

Ploton,D., Menager,M., Deannesson,P., Pigeon,F. and Adnet, J.J. (1986). Improvement in the staining and in the visualization of the argyrophilic proteins of the nucleolar organizer region at the optical level. J.Histochem. 18: 5-14.

Preusser, P.,Wilke, H., Achterrath, W. (1987). Advanced gastric carcinoma . A phase II study with Etoposhide $(\mathrm{E})$, adriamycin $(\mathrm{A})$, and spilit course Cisplatin $(\mathrm{P})=\mathrm{EAP}$. J.Clin.Oncol. 7: 1310-1317.

Pyrhonen, S. (1995). Randomized comparison of 5-fluorouracil, epidoxorubicin, and methotrexate (FEMTX) plus supportive care with supportive care alone in patients with non-resectable gastric cancer. J. Br. Cancer 71: 587-591.

Robbins, B.A., de la Vega,D., Ogata, K.(1987). Immunohistochemical detection of proliferating cell nuclear antigen in solid human malignancies. Arch. Pathol. Lab. Med. 111: 841-845.

Saito A, Korenaga D, Maehara Y. (1991). In vitro succinate dehydrogenase chemosensitivity of gastric carcinoma-relationship to DNA content. Cancer Chemother. Phar. 29: 185-189.

Sasaki K, Ogino T, Takahashi M. (1986). Immunological detection of labeling index on human tumor tissue sections using monoclonal anti-BrdU antibody. Stain Technol. 61: 155-161.

Takahashi Y, Nishioka K. (1995). Survival without tumor shrinkage; Re-evaluation of survival gain by cytostatic effect of chemotherapy. J. Natl. Cancer Inst. 87: 12621263.

Tsuji Y, Suematsu T, Kawabata S, Kodama I, Koufuji K, Aoyagi K., Tanaka T, Maruiwa M, Ohta J, Kumegawa H, Kanda K, Kuboyama S, Adachi T, Takeda J, Kakegawa T. (1994). Complete response in a case of unresectable gastric cancer treated by combined chemoimmunotherapy of MMC, 5-FU and OK-432. Jpn. J. Cancer Chemother. 21: 2045-2048.

Tokimatsu M, Yasutake K, Imamura Y, Ohno S, Okutani T, Matsushita K, Oya M, Yoshimura Y. (1989). A case of partial response of both advanced gastric cancer (Borrmann3) and liver metastasis by treatment with FT-207 sup. The Clinical Report 23: 3936-3940.

Wolly P.V., Smith F, Asteretz R. (1981). A phase trial of 5-FU, adriamycin and Cisplatin (FAP) in advanced gastric cancer. Proc. Amer. Assoc. Cancer Res. 22: 455. 